\title{
Effektivere Behandlung von Bettnässen
}

_Jahrzehntelang dachte man, Bettnässen wachse sich aus. Jedoch wissen wir heute, dass regelmäßiges nächtliches Einnässen ein medizinisches Problem darstellt und relativ leicht behandelt werden kann", sagt Professor Dr. Serdar Tekgül von der urologischen Abteilung der Hacetteppe Universität in Ankara/Türkei. „,Bettnässen betrifft mehr Buben als Mädchen und hat einen starken Einfluss auf das soziale Wohlbefinden. Deshalb würde ich jeder betroffenen Familie dringend raten einen Arzt aufzusuchen."

Bettnässen ist ein häufiges medizinisches Problem [1], das einen beträchtlichen Einfluss auf das Selbstwertgefühl, das emotionale Wohlbefinden und die soziale Entwicklung des Kindes hat sowie auf dessen Tagesablauf (inklusive Leistungen in der Schule; [2, 3, $4,5])$. Bettnässen wird auch mit kognitiven und psychologischen Problemen in Verbindung gebracht, wobei nach einer Behandlung eine Verbesserung der Gedächtnisleistung und anderen täglichen Aktivitäten festgestellt werden konnte [6].

\section{Neue Leitlinie soll Behandlung verbessern}

Die Auswirkungen von Bettnässen werden oft unterschätzt und die Aussowie Weiterbildungen für medizinisches Fachpersonal beinhalten selten spezifische Leitlinien für die Behandlung von Bettnässen. Mit dem Input der World Bedwetting Day Steering Committee-Mitglieder aus aller Welt wurde nun von der European Association of Urology (EAU) die neue Leit-

Weitere

Informationen: www.worldbedwet-

tingday.com

www.clubmond-

kind.at

info@clubmond-

kind.at die im letzten Jahr veröffentlicht wurden [8]. Mit der neuen Leitline, an der auch der österreichische Kinderurologe Univ.-Prof. Dr. Christian Radmayr mitgearbeitet hat, soll nun die Behandlung verbessert und in Folge die Belastung für die Kinder, die durch Bettnässen entsteht, reduziert werden.

„Die Hälfte aller Eltern von betroffenen Kindern im Alter von fünf Jahren oder älter sucht keine ärztliche Hilfe. Sie bevorzugen einen Verbesserungsversuch mit Hilfe von Lebensstiländerungen, und das Kind erfährt erst mit erheblicher Verzögerung medizinische Hilfe - mit all seinen Folgen auf die psychosoziale Entwicklung. Wenn dann ärztliche Hilfe in Anspruch genommen wird, ist es wirklich wichtig, dass sie die richtige Unterstützung und Beratung bekommen", appelliert Tekgül, Chair der neuen Leitlinie.

\section{Neues Video motiviert Eltern zum Handeln}

Das Motto des Internationalen Tag des Bettnässens am 30. Mai lautet Zeit zu handeln. „Das soll Aufforderung sein, dass viel mehr für die Diagnose und effektive Behandlung betroffener Kinder getan werden kann", erklärt Karin Deflorian, Obfrau des österreichischen Vereins Club Mondkind, der Anlaufstelle und Informationsquelle für Familien mit betroffenen Kindern ist. „Bettnässen ist ein häufiges medizinisches Problem, über das Familien ohne Scham und Schuldgefühl mit dem Kinderarzt oder Kinderurologen sprechen sollten." Anstoß kann ein neues Video geben, das das Tabuthema Bettnässen aus dem Mantel des Schweigens holen und betroffene Familien motivieren soll, Hilfe in Anspruch zu nehmen. Zu finden ist das Video im YouTube-Kanal des Vereins Club Mondkind.

\section{World Bedwetting Day Steering Committee}

Das World Bedwetting Day Steering Committee ist eine Arbeitsgruppe, die Experten weltweit zusammenbringt, um diese Initiative zu unterstützen. Sie besteht aus der Internationalen Children's Continence Society (ICCS), der European Society for Paediatric Urology (ESPU), der Asia Pacific

Association (IPNA), der International Paediatric Nephrology Association (IPNA), der European Society of Paediatric Nephrology (ESPN), der Sociedad Iberoamericana de Urologia Paediatrica (SIUP) und der North American Paediatric Urology Societies. Die Initiative wird von Ferring Pharmaceuticals unterstützt.

\section{Literatur}

1. Nevéus T (2011) Nocturnal enuresis-theoretic background and practical guidelines. Pediatr Nephrol 26:1207-1214

2. Bedwetting IV (2017) https://www.verywell. com/bedwetting-bedwetting-statistics-2633257. Zugegriffen: Januar 2017

3. Vande Walle J et al (2012) Practical consensus guidelines for the management of enuresis. Eur J Pediatr 171:971-998

4. Theunis M et al (2002) Self-Image and Performance in Children with Nocturnal Enuresis. Eur Urol 41:660-667

5. Joinson C et al (2007) A United Kingdom population-based study of intellectual capacities in children with and without soiling, daytime wetting, and bed-wetting Pediatrics 120(2):e308-16

6. Van Herzeele C, Dhondt K, Roels SP et al (2016) Desmopressin (melt) therapy in children with monosymptomatic nocturnal enuresis and nocturnal polyuria results in improved neuropsychological functioning and sleep. Pediatr Nephrol. doi:10.1007/ s00467-016-23351-3

7. Tekgül $S$ et al. EAU Guidelines on Paediatric Urology; http://uroweb.org/guideline/ paediatric-urology/?type=pocket-guidelines

8. Oswald J. et al. Leitlinien Kinderurologie; http://www.uro.at/images/uro/downloads/ Leitlinien_Kinderurologie_2016.pdf

Paediatr. Paedolog. Austria 2017 · 52:176

DOI 10.1007/s00608-017-0494-9

(C) Springer-Verlag GmbH Austria 2017 